\title{
The human spongiform encephalopathies
}

\author{
Mavroudis loannis ${ }^{1,2}$, Petrides Foivos ${ }^{2,3}$, Kazis Dimitrios ${ }^{3}$ \\ ${ }^{1}$ Department of Neurology, Leeds, Teaching Hospitals, LTHT, Leeds, UK \\ ${ }^{2}$ Laboratory of Neuropathology and Electron Microscopy, Aristotle University of Thessaloniki, Greece \\ ${ }^{3}$ Third Department of Neurology, Aristotle University of Thessaloniki, Greece
}

\begin{abstract}
The human spongiform encephalopathies are a group of heterogenous, usually fatal diseases, characterized by a unique pathogenetic mechanism and distinct clinical presentation. They are classified into sporadic, familial and acquired forms. The diagnosis of spongiform encephalopathies is based on the combination of the neuropathological examination, with the clinical presentation, the laboratory findings and genetic tests. The most common sporadic form is Creutzfeldt-Jakob disease, while the main familial types are the familial types of Creutzfeldt-Jakob disease, Gerstmann-Straussler-Scheinker disease, Prion disease with cerebral amyloid angiopathy, the fatal familiar insomnia. In the present study we aim to review the main features and types of human spongiform encephalopathies, including the sporadic, familiar, and acquired forms.
\end{abstract}

Keywords: human spongiform encephalopathies, CJD, human prion diseases

\section{INTRODUCTION}

The spongiform encephalopathies are related to pathological forms of prion proteins, and usually characterized by fatal outcome and unique pathogenetic mechanism, in that they can be inherited, they can occur sporadically, or they can be infectious. Prion diseases are neuropathologically characterized by various combinations of spongiform alterations of the brain gray matter, neuronal loss, reactive gliosis, and prion protein deposition. The diagnosis of spongiform encephalopathies can be confirmed with neuropathological examination in combination with clinical presentation, laboratory findings, biochemical and genetic tests (1). The most common form of human prion diseases is Creutzfeldt-Jakob disease with a prevalence of 1-2 cases every 1 million of population, per year, while familial forms are relatively rare, consisting the $10 \%$ of total cases (2). In the present study we aim to review the main features and types of human spongiform encephalopathies, including the sporadic, familiar, and acquired forms.

\section{EPIDEMIOLOGY}

Over the period of 1990-2016 the average annual mortality rates from sporadic Creutzfeldt-Jakob disease (sCJD) in England, Wales, Scotland and Northern Ireland were, respectively, 1.13, 1.40, 1.15 and $0.83 /$ million/year. The mortality rates from $\mathrm{sCJD}$ in the UK are comparable to those observed in most other European countries and elsewhere in the world, including countries that are free of Bovine Spongiform Encephalopathy (BSE). Up to 31 st December 2016, 178 cases of definite or probable variant CJD ( $\mathrm{VCJD}$ ) had been identified in the UK (123 definite and 55 probable cases who did not undergo post mortem) (3).

Two populations are disproportionately affected by CJD: a population of Libyan-born Israelis and some groups in restricted areas of Slovakia where 
the incidence of CJD is 60-100 times greater than expected. These clusters were postulated to be related to dietary exposure of the scrapie agent; but, this was not supported by case-controlled studies, and has been proved that these local high rates of CJD are linked to a high prevalence of codon 200 mutations in the PRNP gene. Prion-related diseases are progressive and invariably fatal with the mean duration of sporadic forms being at 8 months, and familial forms having a mean duration of 26 months, while Gerstmann-Straussler syndrome has the longest course, about 60 months (4).

\section{Prion protein biology}

The transmissible agent in prion diseases has unique features with regards to structure and the remarkable resistance to conventional forms of decontamination. Griffith and Hadlow attempting to identify the agent which was responsible for Scrapie encephalopathy, recognized that the agent was smaller than conventional viruses, did not have RNA or DNA, and could accumulate in the central nervous system $(5,6)$. Prusiner in 1982 stated that the transmissible agent in prion diseases is a protein with a molecular weight of 27 to $30 \mathrm{kD}$, and partially resistant to proteolytic cleavage (7). It was later demonstrated that this protein was a mutated isoform of a protein that normally occurs in the mammalian brain. The normal prion protein is a 253-residue peptide, translated from a single exon within the prion protein gene. The peptide undergoes a series of post-translational modifications, including a cleavage of a signal peptide, and an addition of up to two N-linked oligosaccharide chains at residues 181 and 197 (8). The normal protein contains five octapeptide repeats from codons 51 to 91 while four putative alpha helices in Prion protein are located between codons 109 and 122, 129 and 140,178 and 191, and 202 and 218. Normal prion protein is a membrane-associated protein with short half-life, and is sensitive to proteolytic digestion. Apart from the central nervous system, normal prion protein is expressed in a wide variety of tissues, and although the precise function is not completely understood, recent studies have indicated that it has a role in synaptic function and long-term potentiation (9), might be involved in circadian rhythms, and can act as a copper binding protein, with a protective role in oxidative stress related cell damage (10).
The abnormal isoform of the prion protein accumulates in the central nervous system, has an identical amino acid sequence and the same molecular weight as the normal one, but has a much longer half-life, and is partially resistant to proteolytic digestion (11). Abnormal prion protein has a predominant beta-pleated sheet structure, with loss of the alpha helix regions, and these structural differences confer resistance to proteolytic degradation, and in the same time allow accumulation and aggregation within the central nervous system (12). The mechanisms of neurotoxicity of the abnormal prion protein are not completely understood, however a range of theories exist, from a direct toxic effect on neurons to indirect toxicity mediated by microglia and astrocytes.

\section{HUMAN SPONGIFORM ENCEPHALOPATHIES}

\section{Sporadic Creutzfeldt-Jakob disease}

Sporadic Creutzfeldt-Jakob disease (sCJD) is the most commonly diagnosed human spongiform encephalopathy, consisting about $85 \%$ of total cases, and having a prevalence of 1-2 cases, per million per annum. Both genders are equally affected, and although a wide range of ages at onset has been reported, most cases of sCJD occur in the seventh decade of life (4). sCJD usually presents with rapidly progressive dementia, ataxia, myoclonus, visual impairment, cerebellar syndrome, as well as pyramidal and extrapyramidal signs and symptoms (13). The mean duration of the disease is six months, and the diagnosis can be set, by the combination of clinical presentation, and laboratory tests. The electroencephalogram usually reveals periodic triphasic complexes in $65 \%$ of patients, cerebrospinal fluid analysis shows elevated levels of the chaperone protein 14-3-3, and the brain MRI shows restricted diffusion and hyperintensity on FLAIR sequences (14).

Although the etiology of sCJD is unknown, it has been suggested that this occurs as a consequence of a series of random stochastic events, resulting in the generation or spontaneous conversion of abnormal prion protein within the brain (15). Cohort and case control studies have failed to demonstrate any consistent predisposing factor, however genetic studies have shown that most patient with SCJD are methionine homozygotes at co- 
don 129 , in contrast to normal population, but the significance of this remains uncertain (16). Clinical and neuropathological heterogeneity has given rise to a wide range of syndromes, with the most common and important ones, being the Heidenhain variant with a short clinical history of cortical blindness as a prominent feature, the Brownell - Oppenheimer variant with prominent cerebellar ataxia, the Kuru- plaque variant neuropathologically characterized by amyloid plaques in the cerebellum, the Sporadic fatal familial insomnia, the cortical variant with cortical vacuoles, and the variant CJD with florid and cluster plaques in histopathological examination (17).

\section{Variably protease-sensitive prionopathy}

In 2008, 11 cases of a novel form of prion disease were reported under the term of the variably protease-sensitive prionopathy (VPSPr) in the United States. VPSPr is characterized by behavioral problems and mood changes, language deficits and aphasia, cognitive impairment, and motor signs including parkinsonism. The duration of the disease is usually longer than SCJD, and reaches the 45 months. Neuropathological examination reveals spongiform changes, particularly in the neocortical and subcortical regions of the cerebrum, and spongiform changes and microplaques in the cerebellar cortex. Since the first description of those 11 cases, 19 additional cases have been reported (18-20). The prevalence of VPSPr is low, however there seems to be a relationship between this type of spongiform encephalopathy and $129 \mathrm{VV}$ genotype, although some patients of the PRNP codon 129MM and $129 \mathrm{MV}$ genotypes have been reported. Subsequent studies showed differences between 3 codon 129 genotypes and protease sensitivity of the abnormal prion protein $(21,22)$.

\section{FAMILIAL FORMS OF SPONGIFORM ENCEPHALOPATHIES}

Familial spongiform encephalopathies are inherited with the autosomal dominant character and high penetrance, and include familial CreutzfeldtJakob disease (fCJD), Gerstmann-StrausslerScheinker disease (GSS), variable phenotypes, and fatal familial insomnia (FFI). The first mutation to be identified in familial spongiform encephalopathies was the PRNP P102L in 1989, and since then numerous further mutations have been described, all affecting the octapeptide repeat region, and each one of them strongly related to the clinical and pathological features of the disease (17).

\section{Familial CJD}

Familial CJD's clinical and neuropathological features resemble the sporadic form, however PRNP gene mutations are found with the most common being the E200K-129M and D178-129V. Familial CJD is characterized by the isotype $1 \mathrm{~B}$ of abnormal prion protein with cortical or diffuse spongiform degeneration, and isotype $2 \mathrm{~B}$ with diffuse cortical spongiform degeneration and focal cerebellar plaques (23).

\section{Gerstmann-Straussler-Scheinker disease}

Gerstmann-Straussler-Scheinker disease is characterized by a cerebellar syndrome accompanied by pyramidal signs and progressive cognitive impairment usually leading to dementia. The neuropathological spectrum of the disease consists of multicentric PrP-amyloid plaques in the cerebral and cerebellar cortex, with or without spongiform changes. A number of point mutations resulting in a similar clinicopathological phenotype has been described, however mutations F198S and Q217R are associated with neocortical neurofibrillary tangles in addition to widespread multicentric and unicentric prion protein - amyloid plaques (24).

\section{Prion disease with cerebral amyloid angiopathy (PRP-CAA)}

PRP-CAA is characterized by selective vascular wall accumulation of PrP-amyloid deposits, and is related to rare stop mutations in PRNP gene. The clinical course is usually a relatively prolonged progressive cognitive decline leading to dementia, while the common histological features are vascular and perivascular amyloid deposits, minimal spongiform change, and tangle accumulation in neurons near the affected vessels (25).

\section{Prion disease associated with octapeptide repeat region insertional mutations (variable pheno- types)}

The clinical manifestations of this group of prion diseases are highly variable both in terms of dis- 
ease duration, and in phenotype. Patients with up to four additional copies of the octapeptide repeats, have a clinical phenotype similar to sporadic CJD with rapidly progressive dementia and similar electroencephalographic findings. Patients with larger numbers of extra repeats, present with a more variable clinical phenotype, often with ataxia and other movement disorders. Neuropathological examination shows unusual linear prion protein deposits in the molecular layer of the cerebellum, and somewhat variable histological features (26).

\section{Fatal familiar insomnia}

Fatal familiar insomnia is characterized by sleep disturbance, dysautonomia, motor signs and mild progressive cognitive abnormalities. The onset of the disease varies between 18 and 60 years and the mean disease duration from 8 months to 3 years. It is noteworthy that in Basconia a number of cases of fatal familial insomnia with catatonic features have been described (27). From the neuropathological point of view the disease is characterized by severe neuronal loss and gliosis in the anterior thalamic nuclei and in the hypothalamus, in the inferior olivary nuclei, and to a lesser extent in the cerebral and cerebellar cortex, and the absence of spongiform change or abnormal prion protein deposition. In some cases, tissue blots obtained from sections of paraffin-embedded tissue may show abnormal prion protein deposition in the entorhinal cortex. Fatal familiar insomnia is caused by a mutation in codon 178 at the PRNP gene (17).

\section{ACQUIRED PRION DISEASES}

\section{Kuru encephalopathy}

Kuru encephalopathy was described among the Fore tribe of Papua New Guinea in the 1950s and is characterized by progressive ataxia and tremor with marked emotional instability Rapidly progressive dementia was not a common feature. The Fore people ritualistically cooked and consumed body parts of their family members following their death to symbolize respect and mourning. Because the brain is the organ enriched in the infectious agent prion, women and children, who consumed brain and viscera, had much higher likelihood of being infected than men, who preferentially consumed muscles (28). The disease was associated with ritu- alistic cannibalism, and now extinct, with some of last symptomatic patients sustained incubation periods of around 40 years (29). Neuropathological examination revealed amyloid plaques, so-called Kuru plaques in the cerebellum and particularly in the granular layer, and spongiform changes in the cerebellum, the basal ganglia, and the thalamus, with a variable distribution in the cerebral cortex (30).

\section{latrogenic CJD}

The first case of iatrogenic CJD was reported in 1974 in a patient who had a corneal transplant, and since then more than 400 cases have been identified, the majority of which have been recipients of autopsy-derived human pituitary hormones or human dura mater grafts. The clinical features are variable, ranging from a similar to sporadic CJD presentation, to a progressive cerebellar ataxia, focal neurological symptoms and dementia. The clinical presentation and incubation times are related to the route of infection. The central route is characterized by a shortest incubation period, and the peripheral route has a longer incubation period (31). The neuropathological examination reveals findings similar to the sporadic CJD (17).

\section{Variant CJD}

In 1996, a novel form of prion disease with unusual clinical, biological, and pathological features was identified by the National CJD Surveillance Unit in the United Kingdom, and Up to 31st December 2016, 178 cases of definite or probable vCJD had been identified in the UK (32). 58\% of cases were males and $42 \%$ females. The median age at onset was $261 / 2$ years and the median age at death 28 years. The youngest case was aged 12 years at onset while the oldest case was aged 74 years. The median duration of illness from the onset of first symptoms to death was 14 months, ranging from 6 to 114 months. A causative relationship between VCJD and the epidemic of bovine spongiform encephalopathy in cattle seemed likely. The clinical presentation includes psychiatric and/or sensory manifestations at onset, followed by severe progressive ataxia, extrapyramidal and pyramidal signs, and a progressive dementia, which in some cases was severe (33). The EEG is abnormal, however it does not show the characteristic abnormalities seen in sporadic CJD, and brain MRI shows 
symmetrical areas of hyperintensity on FLAIR sequences in the posterior thalamus, the so-called pulvinar sign, which is highly characteristic and has been incorporated into the diagnostic criteria (34). CSF analysis may exhibit elevated phosphorylated tau, and the neuropathological examination shows large numbers of florid plaques with a widespread distribution in the cerebral cortex and in the cerebellum. The lesions comprise of a central eosinophilic amyloid core with radiating bundles of amyloid fibrils, surrounded by spongiform chang- es. Other neuropathological features include extensive abnormal prion protein accumulation both in small cluster plaques and diffuse deposits, with spongiform changes most marked in the caudate nucleus and putamen, and extensive neuronal loss and gliosis in the posterior thalamic nuclei. Western blot analysis has shown a characteristic abnormal prion protein isotype with a glycosylation pattern similar to that seen on Western blot analysis for abnormal prion protein in cattle with Bovine Spongiform Encephalopathy (17).

TABLE 1. Sporadic CJD diagnostic criteria (Adapted from: a) Global Surveillance, diagnosis, and Therapy of Human Transmissible spongiform Encephalopathies: Report of WHO consultation, February 9-11, 1998, Geneva, Switzerland; and b) Zerr, I, Kallenberg K, Summers DM, et al. Brain 2009, 132; 2659-2668.)

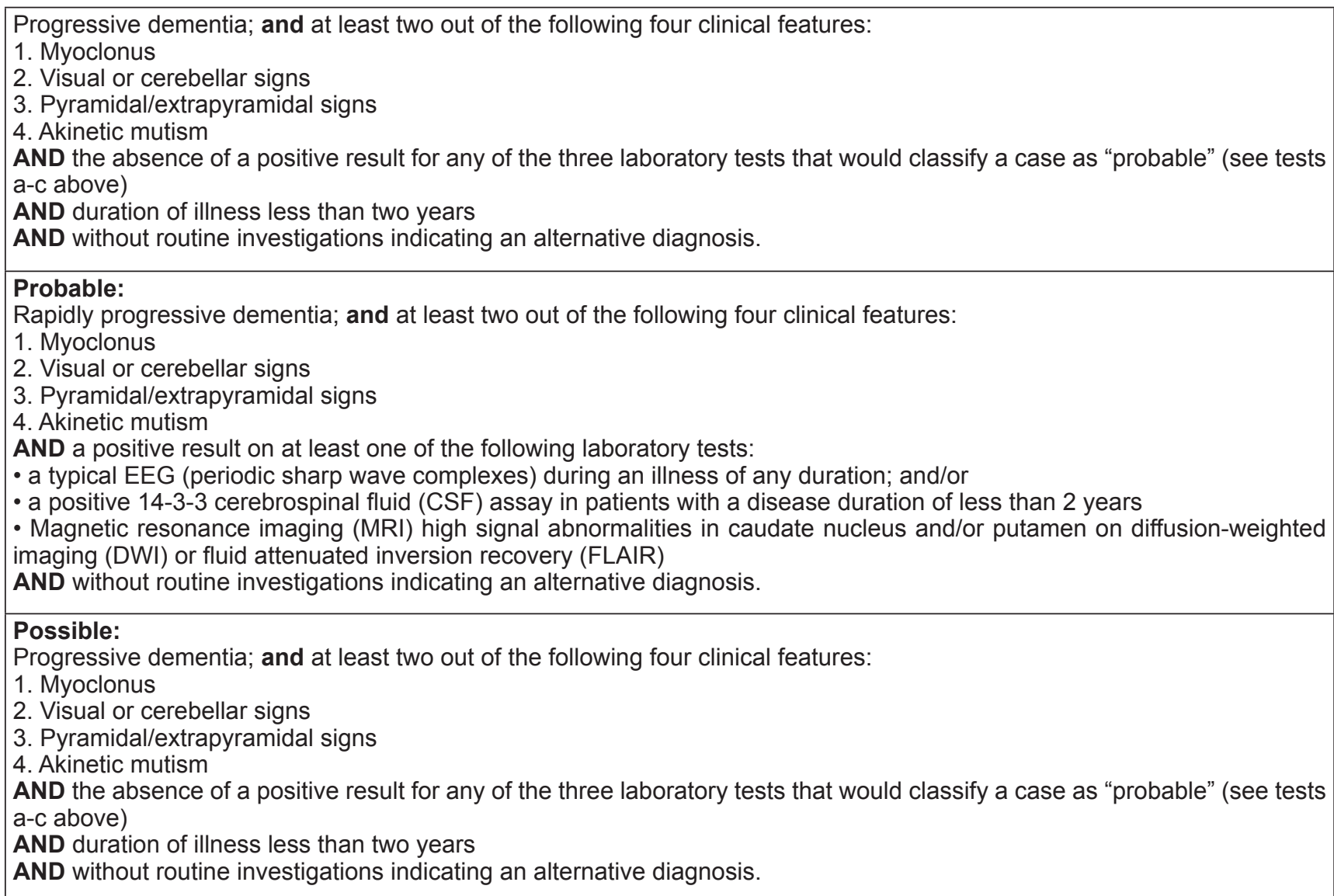

TABLE 2. Iatrogenic CJD diagnostic criteria (Adapted from: a) Global Surveillance, diagnosis, and Therapy of Human Transmissible spongiform Encephalopathies: Report of WHO consultation, February 9-11, 1998, Geneva, Switzerland; and b) Zerr, I, Kallenberg K, Summers DM, et al. Brain 2009, 132; 2659-2668.)

\footnotetext{
Progressive cerebellar syndrome in a recipient of human cadaveric-derived pituitary hormone; Or sporadic CJD with a recognized exposure risk, e.g., antecedent neurosurgery with dura mater implantation.
} 
TABLE 3. Familial CJD diagnostic criteria (Adapted from: a) Global Surveillance, diagnosis, and Therapy of Human Transmissible spongiform Encephalopathies: Report of WHO consultation, February 9-11, 1998, Geneva, Switzerland; and b) Zerr, I, Kallenberg K, Summers DM, et al. Brain 2009, 132; 2659-2668.)

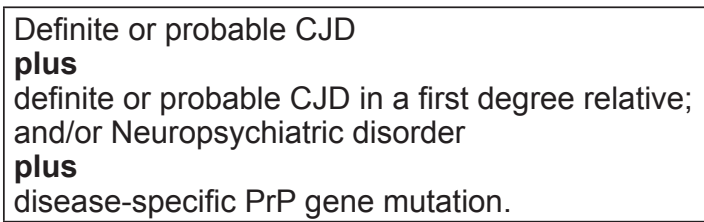

\section{REFERENCES}

1. Takada L.T., Geschwind M.D. Prion diseases. Semin Neurol. 2013 Sep;33(4):348-56. doi: 10.1055/s-0033-1359314. Epub 2013 Nov 14

2. Sadowski M., Pankiewicz J., Scholtzova H., Ripellino J.A., Li Y., Schmidt S.D., Mathews P.M., Fryer J.D., Holtzman D.M., Sigurdsson E.M., Wisniewski T. A synthetic peptide blocking the apolipoprotein E/beta-amyloid binding mitigates beta-amyloid toxicity and fibril formation in vitro and reduces beta-amyloid plaques in transgenic mice. Am J Pathol. 2004 Sep;165(3):937-48.

3. The National CJD Research \& Surveillance Unit Western General Hospital, Edinburgh, EH4 2XU 25th ANNUAL REPORT 2016 Creutzfeldt-Jakob disease, Survellance in the UK www.cjd.ed.ac.uk

4. Masters C.L., J.O. Harris, D.C. Gajdusek, C.J. Jr Gibbs, C. Bernoulli, D.M. Asher. Creutzfeldt-Jakob disease: patterns of worldwide occurrence and the significance of familial and sporadic clustering. Ann Neurol., 1979 : 5(2):177-88.

5. Griffith J.S. Self-replication and scrapie. Nature. 1967; 215:1043-4.

6. Hadlow W.J. Scrapie and kuru. Lancet. 1959; 2:289-90.

7. Prusiner S.B. Novel proteinaceous infectious particles cause scrapie. Science, 1982: 216(4542):136-44.

8. Prusiner S.B. Neurodegeneration in humans caused by prions. West J Med. 1994 Sep; 161(3): 264-272.

9. Collinge J. Molecular neurology of prion disease J Neurol Neurosurg Psychiatry. 2005 Jul; 76(7): 906-919.

10. Weissmann C., Fischer M., Raeber A., Büeler H., Sailer A., Shmerling D., Rülicke T., Brandner S., Aguzzi A. The use of transgenic mice in the investigation of transmissible spongiform encephalopathies. Int J Exp Pathol. 1996 Dec; 77(6): 283-293.

11. James T.L. et al. Solution structure of a 142-residue recombinant prion protein corresponding to the infectious fragment of the scrapie isoform. Proc Natl Acad Sci USA, 1997: 94(19):10086-91

12. Riek R., Hornemann S., Wider G., Billeter M., Glockshuber R., Wuthrich K. NMR structure of the mouse prion protein domain PrP(121-321). Nature, 1996: 382(6587):180-2

13. Murray E.D., N. Buttner B.H. Price. Depression and Psychosis in Neurological Practice. In Neurology in Clinical Practice, 6th Edition, by WG Bradley, RB Daroff, GM Fenichel and J (eds.) Jankovic. Oxford: Butterworth Heinemann, 2012

14. Chiofalo N., Fuentes A., Galvez S. Serial EEG findings in 27 cases of Creutzfeldt-Jakob disease. Arch. Neurol., 1980, 37: 143-145.

15. Prusiner S.B., M.R. Scott, S.J. DeArmond, F.E. Cohen. Prion protein biology. Cell, 1998: 93(3):337-48

16. Thackray A.M., Knight R., Haswell S.J., R. Bujdoso D.R. Brown. Metal imbalance and compromised antioxidant function are early changes in prion disease. Biochem J, 2002: 362:253-8

17. Gray F., Duyckaerts C., De Girolami U. Escourolle and Poirier's Manual of Basic Neuropathology (5 ed.). Oxford University Press, 2013

18. Head M.W., Knight R., Zeidler M., Yull H., Barlow A., Ironside J.W. A case of protease sensitive prionopathy in a patient in the UK. Neuropathol Appl Neurobiol. 2009; 35:628-32.

19. Jansen C., Head M.W., van Gool W.A., Baas F., Yull H., Ironside J.W. The first case of protease-sensitive prionopathy (PSPr) in the
Netherlands: a patient with an unusual GSS-like clinical phenotype. J Neurol Neurosurg Psychiatry. 2010; 81:1052-5.

20. Rodríguez-Martínez A.B., Garrido J.M., Zarranz J.J., Arteagoitia J.M., de Pancorbo M.M., Atares B. A novel form of human disease with a protease-sensitive prion protein and heterozygosity methionine/valine at codon 129: case report. BMC Neurol. 2010;10:99.

21. Zou W.Q., Puoti G., Xiao X., Yuan J., Qing L., Cali I. Variably protease-sensitive prionopathy: a new sporadic disease of the prion protein. Ann Neurol. 2010;68:162-72

22. Diack A. et al. Variably Protease-Sensitive Prionopathy, a Unique Prion Variant with Inefficient Transmission Properties. Emerg Infect Dis., 2014: 20(12):1969-1979

23. Goldfarb L.G. et al. Creutzfeldt-Jakob disease cosegregates with the codon 178Asn PRNP mutation in families of European origin. Ann Neurol, 1992: 31(3):274-81

24. Ghetti B., Tagliavini F., Giaccone G., Bugiani O., Frangione B., Farlow M.R., Dlouhy S.R. Familial Gerstmann-Sträussler-Scheinker disease with neurofibrillary tangles. Mol Neurobiol., 1994: 8(1):41-8

25. Ghetti B., Piccardo P., Spillantini M.G. et al. Vascular variant of prion protein cerebral amyloidosis with tau-positive neurofibrillary tangles: the phenotype of the stop codon 145 mutation in PRNP. Proceedings of the National Academy of Sciences of the United States of America. 1996; 93(2):744-748.

26. Moore R.A., Herzog C., Errett J., Kocisko D.A., Arnold K.M., Hayes S.F., Priola S.A. Octapeptide repeat insertions increase the rate of protease-resistant prion protein formation. Protein Sci. 2006 Mar; 15(3):609-19. Epub 2006 Feb 1

27. Oliveros R.G., N. Saracibar, M. Gutierrez, T. Munon, A. GonzalezPinto. Catatonia due to a prion familial disease. Schizophr Res, 2009: 108(1-3):309-10

28. Haïk S., Brandel J.P. Infectious prion diseases in humans: Cannibalism, iatrogenicity and zoonoses. Infect Genet Evol. 2014 Aug. 26C:303-312

29. Liberski P.P. et al. Kuru: genes, cannibals and neuropathology. J Neuropathol Exp Neurol., 2012: 71(2):92-103

30. Imran, Muhammad; Mahmood, Saqib (2011-01-01). "An overview of human prion diseases". Virology Journal. 8: 559

31. Collinge J., Poulter M., Davis M.B., Baraitser M., Owen F., Crow T.J., Harding A.E. Presymptomatic detection or exclusion of prion protein gene defects in families with inherited prion diseases. Am J Hum Genet. 1991 Dec; 49(6): 1351-1354.

32. Collinge J., Sidle K.C., Meads J., Ironside J., Hill A.F. Molecular analysis of prion strain variation and the aetiology of 'new variant' CJD. Nature. 1996 Oct 24; 383(6602):685-90.

33. Bateman D.D. Hilton, S. Love, M. Zeidler, J. Beck, J. Collinge. Sporadic Creutzfeldt-Jakob disease in a 18-year-old in the UK. Lancet, 1995: 346(8983):1155-6

34. Geoffrey Y. et al. Diffusion-Weighted and Fluid-Attenuated Inversion Recovery Imaging in Creutzfeldt-Jakob Disease: High Sensitivity and Specificity for Diagnosis. American Journal of Neuroradiology, 2005: 26(6): 1551-1562 